\title{
The evolution and transformation of telemedicine
}

\section{What is telemedicine?}

Telemedicine also referred to as "telehealth" or "e-health"), was introduced in 1970s for the first time. It means the use of telecommunication and information technology to provide clinical health care from a distance. ${ }^{1}$ In spite of numerous studies, unfortunately, there is no integrated definition of telemedicine. The definition of the term telemedicine as accepted by World. ${ }^{2}$ Health Organization is, "The delivery of health care services, where distance is a critical factor, by all health care professionals using information and communication technologies for the exchange of valid information for diagnosis, treatment and prevention of disease and injuries, research and evaluation, and for the continuing education of health care providers, all in the interests of advancing the health of individuals and their communities. ${ }^{3}$

\section{Origins and history}

Telemedicine history goes back to the nineteenth century. According to this history, this phrase was introduced with one of the first reports published in the twentieth century, ${ }^{4}$ at which time electrocardiography data was transmitted over telephone wires. ${ }^{5}$ Commercial equipment for the development of telemedicine in the 1960 s was used by the military and space technology departments as well as private individuals. ${ }^{6}$ The use of TV to facilitate relationship between specialists at a psychiatric institute and general practitioners at a state mental hospital, ${ }^{7}$ and providing medical advice from a major teaching hospital to an airport medical center can be noted as some examples of early technological milestones in telemedicine. ${ }^{8}$ Many parameters have been described as drivers of telemedicine over the past decade, the biggest ones include recent advancements in, and increasing availability and utilization of, ICTs by the general population, rapidly creating new possibilities for health care service and delivery. Developing countries and disadvantaged areas of industrialized nations follows this. ${ }^{9}$ The use of telemedicine among health-care providers has been increased due to the replacement of analogue forms of communication with digital methods, combined with a rapid drop in the cost of ICTs. These have made health care organizations enable to imagine as a future possibility and use new and more efficient methods of providing care. The speed of the advancement of communication and information technology has increased with the introduction of the Internet, after which the telemedicine domain has expanded into web based applications and multimedia approaches. The creation of a tapestry rich in telemedicine programs is the result of these advances that can be used in various parts of the world.

\section{Ancient times}

According to available evidence, for communicate of medical information, some communication intermediaries have been used, especially smoke signals and light reflections. Telecommunication methods have been used to indicate the prevalence of infections and to inform about health events such as birth or death. In this way, smoke signals were used to indicate the occurrence of medical and health incidents by American Indian tribes in ancient Greece.

\section{Early telemedicine}

It has been reported that modern telemedicine began by the

\author{
Volume 3 Issue 4 - 2017
}

\author{
Hamidreza Shirzadfar, Fatemeh Lotfi \\ Department of Biomedical Engineering, Sheikhbahaee University, \\ Iran
}

Correspondence: Hamidreza Shirzadfar, Department of Biomedical Engineering, Sheikhbahaee University, Baharestan, Isfahan, Iran, Email: h.shirzadfar@shbu.ac.ir

Received: July 21, 2017 | Published: October 30, 2017

inventions of the electrical telegraph and the telephone. Despite the introduction of other inventions, such as flag semaphores and light telegraph, they were primarily used for military and naval communication. The scope of telemedicine beyond ancient times were not developed communication inventions before the telegraph and telephone. Mail, another popular long-distance communication medium, was used for medical communication. However, from a technological perspective, mail can be considered simply an extension of the human messenger. Long distance communication is brought into the mainstream by the telegraph and telephone so that almost anyone are able to send a telegraph message or make a phone call. Telegraph was considered as a special-use technology and since special training was needed to work with the telegraph, and there was not enough network to receive the telegraph, few people installed telegraph equipment in their homes. However, given the high telegraph communication speed, the technology was used for telemedicine in military situations. Gradually the use of the telegraph was developed, so that it was used for ordering medical supplies as well as communicating deaths and injuries on the battlefield during the U.S. Civil War. It has reported that telegraph was also used for medical consultations. Creating a connected world made possible by telephone. Telephones in hospitals and doctors' offices were installed in major cities. Also residents of many cities within a few years, installed the phone in their homes. A huge transformation emerged in the medical world with the phone, so that patients could talk to all of a sudden physician over the telephone and receive medical advice directly. Also, in order to consult or exchange information, medical providers could speak over the phone to other physicians.

In the modern work, all of children consider the telephone as a necessary tool. Telephones are used from our childhood. The use of telephone is not considered as a telemedicine application by many people purposely. When patients discuss their blood test results with a nurse over the phone, don't consider that it is unusual. According to the studies, phone usage is declining. Now, other asynchronous communication methods, such as text messages are preferred by many people, because they are more convenient for them. It should be noted that the telephone is considered as the foundation for many later communication and telemedicine technologies. It has reported that the overall application of the telephone was increased through the 1900 s as a significant increase can be seen in telephone network with higher quality signals, telephone numbers, and other features. In the United States, 9-1-1 was introduced as the official emergency telephone number in 1968. Some applications of this number includes: report a fire, get the police, or a medical emergency. Earlier on, using the telephone people had to get the operator to forward their call to 
the right department (police, fire station, or hospital), or to know the specific number to dial. According to the telemedicine perspective, a consistent and faster access to emergency medical care was provided by 9-1-1. Although, Telefax was invented before the telephone did not electric printing telegraph gain significant traction until common telephone lines and telephone numbers for transmissions were used by fax devices. Up to now, faxes are used by medical professionals use in order to transmit medical records.

\section{Rise of telemedicine}

As we know, for the first time, telemedicine was introduced in the April 1924 issue of Radio News magazine. The magazine described that how a patient can communicate with a doctor, including use of heartbeat and temperature indicators. An imagination of the future was formed the basis of the concept, as U.S. residents did not yet have televisions in their homes, and radio adoption was just gaining steam.

In the early 1900s, suggestions were made for the transmission of data related to stethoscope and other devices through communication channels (telephone, radio, etc.). Nevertheless, none of these experiments did not succeed. It has reported that for the first time telemedicine to transmit video, images, and complex medical data were used in the late 1950s and early 1960s. In 1959, in order to transmit neurological examinations, interactive telemedicine was used by the University of Nebraska, which is widely considered the first case of a real-time video telemedicine consultation. Other programs often used in an academic setting, which focused on transmission of medical data such as fluoroscopy images, x-rays, stethoscope sound, and electrocardiograms (ECGs). These early projects were carried aimed to achieve the following:

i. Providing access to health care in rural areas.

ii. Urban medical emergency situations.

According to studies, there was a major break for the progress of telemedicine in 1960s when several partners decided to join together to work on STARPAHC project, these partners include the National Aeronautics and Space Administration (NASA), Lockheed Corporation, and U.S. Indian Health Service. Space Technology Applied to Rural Papago Advanced Health Care (STARPAHC), is considered as a large-scale telemedicine project. Telemedicine access was provided to an American Indian reservation using the same technologies intended for astronauts on space missions. Many initiatives in relation with grant and government-supported telemedicine have been reported, including. ${ }^{10}$

i. Providing medical care in a war zone.

ii. Providing medical care to remote scientific stations in Arctic and Antarctic.

iii. Providing medical care to correctional facilities without transporting inmates to the hospital.

iv. Digital transmission of radiology images.

The first medical specialty to fully embrace telemedicine was Radiology. The reliability and efficiency of telemedicine was proved with the help from grant-sponsored projects, teleradiology has won the trust of the medical community. Some radiologists decided to use teleradiology systems to receive images for telemedicine consultations in 1980s. According to studies, large undertakings requiring considerable staff and organizational changes were involved in the telemedicine projects in almost all of the early deployments of telemedicine. Custom hardware and software equipment were used to implement the telemedicine implementations. Only trained persons could use the bulky equipment, so that the average patient did not directly interact with telemedicine technologies. Instead, a telepresenter handled the equipment and interacted with the patient. Original forms of many early projects have changed due to the advancement of technology and other factors. Telemedicine refer to these early types of telemedicine deployments. The following can be used to describe these projects:

a. Custom, large hardware which is created only for telemedicine.

b. Designed for specific applications, such as psychiatry consults in ER.

c. Expensive.

d. Need for specially trained tele presenters.

\section{Telemedicine and the internet}

Information explosion is one of the results of the rise of the Internet in the 1990s. Support of all information and traffic needed for telemedicine became possible using the Internet protocols, the information includes:

i. Educational levels of patient (text, images, video).

ii. Medical images such as x-rays and scans (DICOM image standards).

iii. Real-time audio and video consultation.

iv. Vital signs and other body measurements (ECG, temperature, etc.).

Globalization, content publishing, consumer demand, and other factors outside of health care drove the Internet growth. This growth meant that considerable funds and engineering efforts went into Internet infrastructure improvements, including:

i. Communication speeds (bandwidth and latency).

ii. Storage of information (databases, object-store for large files such as images and video).

iii. Accessibility many web services employ backup servers, and even dynamically start up additional servers if traffic increases.

iv. Standard formats to transmit data (MP4, PNG, etc.).

v. Security (encryption, password protection, access levels, etc.).

vi. Application development new programming languages (JavaScript), frameworks, and open-source software (Apache).

vii. The Cloud using virtual servers hosted by an infrastructure provider such as Amazon Web Services (AWS).

viii. Digitizing information (digital cameras, scanners, etc.).

Health care and telemedicine was affected by the above Internet improvements. It was easier and cheaper than ever to build a health care software application for exchanging and storing clinical data, using the existing tools and frameworks for web applications. The U.S. government incentives (and future penalties) as leader has opened the e-health floodgates opened with the transition to electronic medical records (EMRs. The Internet is used by most of today's 
modern EMR vendors to make possible access to medical information for medical providers and patients. Moreover, the use of this patient portals has increased, where patients can look up their lab results, refill prescriptions, or send a secure message to their physician. The knowledge of both medical providers and patients is increasing. The Internet is now widely used in health care -it is surprising to hear of a private practice without a web site. Many efforts are made by practices to leverage the Internet further by engaging existing and potential new patients through social media outlets like Twitter and Facebook. Also, they access their patients and encourage them to post reviews on Yelp and other web sites. Also, access to tons of medical information online has been possible for patients. Many patients research their symptoms using "Doctor Google" before coming to see their physician. ${ }^{11}$ Of course, reliability is one important issue in relation to get information from public sources on the Internet. Unfortunately, some articles provide inaccurate information for patients.

Today, the Internet is widely used in day-to-day life. Accessing the Internet became possible in mobile device such as a smart-phone or tablet and often U.S. adults are using it. These devices are used often as their primary entry point for the online world. Some key factors in the ongoing Telemedicine transition include: the ubiquity of the Internet, the ready access to Internet-enabled computing devices, and the technical savvy of the U.S. population. The term suffix is used to refer applications running on Web technologies, and characterized by their collaboration, usability, interoperation, and openness features. One of the most important applications of the moniker is health care. The following can be used to describe Telemedicine 2.0:

i. The use of computing device available belonging to patient or physician.

ii. Establishment communication over the Internet and the use of standard web infrastructure.

iii. The use of cheap off-the shelf equipment to collect clinical data.

iv. It can be used easily and directly by patient or physician without special training.

There are affordable measurements devices that are widely used with telemedicine include:
i. Smartphone cameras.
ii. Digital stethoscopes
iii. Ophthalmoscopes (for eye exams).
iv. Otoscopes (for ear exams).
v. Vital sign monitoring devices.
vi. Wearable biosensors.
vii. Microstrip patch Antenna.

Over time, Telemedicine has been successful to full cover the Internet communication medium. Many changes have been made in private practices and health care systems. These systems are in the process of becoming hybrid health care providers allowing patients to see their medical provider either through telemedicine or in-person. We examine the categories and types of Telemedicine use cases in the Categories of Telemedicine article. At the present time, one of the most popular types of printed antenna is microstrip antenna. It has many advantages. In telemedicine, different forms of microstrip patch antenna can be used such as square, rectangle, circular and triangle. ${ }^{12}$ Metallic patch and ground plane are two main components of the microstrip antenna (Figure 1). The ATS-1 Satellite was launched on December 7th, 1966 by NASA (Figure 2), it was the first satellite used for civic purposes, such as education, and telemedicine. This is considered as one of the first of many telemedical satellite projects which were conducted by NASA over the next 50 years. New and expanded opportunities were provided by the ATS-6 for testing a variety of new space communications concepts which need to use geostationary spacecraft. Up to that time, it was the most technologically advanced communications satellite in terms of its capacity, power, and precision. A new opportunity was provided for telemedicine based on the amount of bandwidth offered by NASA to telemedical facilities. Figure 3 shows the graphic of ATS-6.
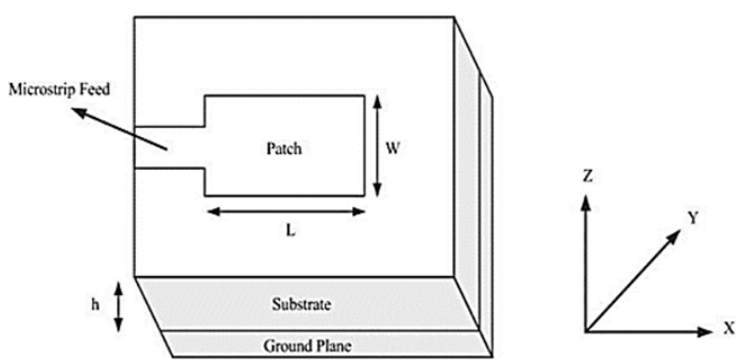

Figure I Schematic of a micro strip patch antenna. ${ }^{13}$

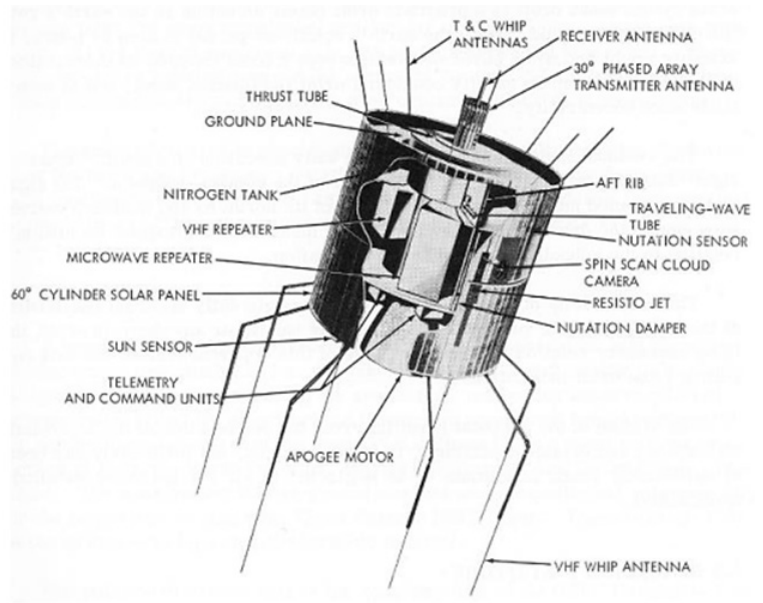

Figure 2 The Schematic of ATS-I Satellite. ${ }^{14}$

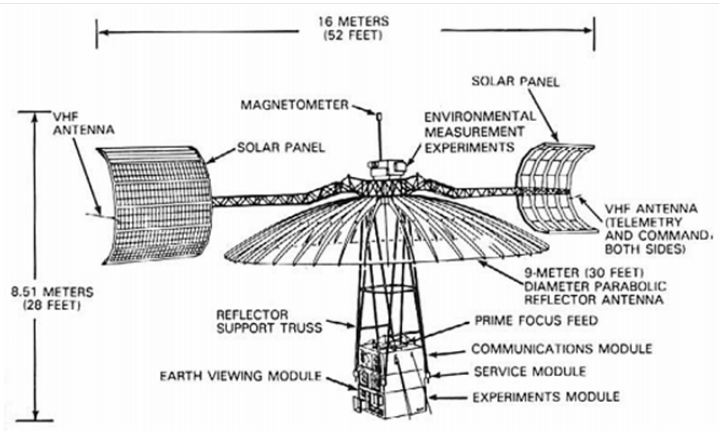

Figure 3 The launch of ATS-6 in 1974 ushered in a new era for satellite- based telemedicine experimentation [15]. 


\section{Conclusion}

This study was carried out aimed to describe the history of telemedicine. Telemedicine faced with many ups and down to make significant change in tools by development of telecommunications from electrocardiograph to satellite in order to promote the cure of the human. Nowadays, we are seeing competition between telemedicine with internet to have more significant impact on health of the world. Moreover, the history of telemedicine is briefly studied. For future studies, we want to study telemedicine devices and also the micro strip patch antenna. ${ }^{13-15}$

\section{Acknowledgements}

None.

\section{Conflict of Interest}

The author declares no conflict of interest.

\section{References}

1. Strehle EM, Shabde N. One hundred years of telemedicine: does this new technology have a place in paediatrics? Archives of disease in childhood. 2006;19(12):956-959.

2. Sood SP, Negash S, Mbarika VW, et al. Differences in public and private sector adoption of telemedicine: Indian case study for sectoral adoption. Stud Health Technol Inform. 2007;130:257-268.

3. A Health Telematics policy in support of WHO'S health-for-all strategy for global development: report of the WHO group consultation on health telematics. Switzerland: WHO; 1998. 38 p.

4. Craig J, Patterson V. Introduction to the practice of telemedicine. Journal of telemedicine and telecare. 2005;1(1):3-9.
5. Einthoven W. Le télécardiogramme. Arch Intern Physiol. 1906;4:132164.

6. Currell R, Urquhart C, Wainwright $\mathrm{P}$, et al. Telemedicine versus face to face patient care: effects on professional practice and health care outcomes. Cochrane Database Syst Rev. 2000;2:CD002098.

7. Benschoter RA, Eaton MT, Smith P. Use of videotape to provide individual instruction in techniques of psychotherapy. J Med Educ. 1965;40(12):1159-1161.

8. Dwyer TF. Telepsychiatry: psychiatric consultation by interactive television. American Journal of Psychiatry.1973;130(8):865-869.

9. Wootton R, Jebamani LS, Dow SA. E-health and the Universitas 21 organization: 2. Telemedicine and underserved populations. Journal of Telemedicine and Telecare. 2005;11(5):221-224.

10. History of telemedicine.

11. Leune AS, Nizard J. Doctor Google: use of Internet during pregnancy in France in 2009. J Gynecol Obstet Biol Reprod (Paris). 2012;41(3):243254.

12. Shirzadfar H, Shirvani P. Design a novel microstrip rectangle patch antenna utilized in telemedicine applications. $J$ Bioanal Biomed. 2016;8(5):e145.

13. Shirvani P, Shirzadfar H. Design a new configuration of micro strip rectangle patch antenna on different thickness substrate for telemedicine applications. Journal of Nano-and Electronic Physics. 2016;8(3):30283031.

14. ATS Image Collection.

15. Spacecrafts launched in 1974. 